\title{
New Oxygenation Method Based on Pulse Oximeter
}

\author{
Soroor Behbahani ${ }^{1, *}$, Mohammad Ali Pishbin ${ }^{2}$ \\ ${ }^{1}$ Department of Engineering, Islamic Azad University, Garmsar Branch, Garmsar, Iran \\ ${ }^{2}$ Department of Biomedical Engineering, Islamic Azad University, Science Research Branch, Tehran, Iran
}

\begin{abstract}
The body's need of oxygen is certainbecause of its necessity to make energy from the food. Lungs deliver oxygen to the blood and if it cannot get enough oxygen to the blood, the patient feels short of breath, which is very uncomfortable. In this state, oxygen therapy can be helpful. Blood gas measurement provides vital information about oxygenation, ventilation and acid-base status. However, these measurements only provide a snapshot of the patient's condition taken at the time that blood sample was drawn. Oxygen level can change very quickly. In the absence of continuous oxygenation monitoring, these changes may go undetected until it is too late. In this paper, we present a new method of online oxygenation, which, could give oxygen to the patient with high accuracy and solve many problems involved in other methods. Ten patients; five COPD and five patients suffer of asthmawere examined with this new method of oxygenation. None of the patients exhibited signs of oxygen toxicity during new method of oxygen therapy. Experimental results show the acceptability of new oxygenation method in examined subjects.
\end{abstract}

Keywords Oxygen, Pulse Oximeter, Oxygenation

\section{Introduction}

Oxygen is necessary for the body because all of the main organs and tissues use it. When the body does not get enough oxygen, the patient may have difficulty breathing, problems with heart, fatigue, loss of memory, headaches, or confusion.

Oxygen therapy provides extra oxygen, which the body needs to work well. Normally, lungs absorb oxygen from the air. However, some diseases and conditions may prevent patient from getting enough oxygen. Oxygen therapy can increase the supply of oxygen to the lungs and thereby increasing the availability of oxygen to the body. Usually oxygen flows through a tube and delivered to the lungs through a nasal cannula, a facemask or a s mall tube inserted into windpipe through the front of patient neck.

Oxygen therapy can be done in a hospital, medical setting, or even at home. If the patient needs oxygen therapy for an ongoing disease or condition, he/she might receive home oxygen therapy. The oxygen is prescribed to treat a number of conditions regard lung and heart diseases such as chronic obstructive pulmonary disease (COPD), chronic bronchitis, emphysema, severe asthma and hypertension.It may be pres cribe to treat a group of patients who will require oxygen for palliative care or short burst needs[1].

Patients, who suffer from COPD, rely on oxygen tanks and concentrators to deliver a more pure form of oxygen so that they get the right amount to their blood cells.

\footnotetext{
* Corresponding author:

Sor.behbahani@gmail.com (Soroor Behbahani)

Published online at http://journal.sapub.org/ajbe

Copyright (C) 2012 Scientific \& Academic Publishing. All Rights Reserved
}

There are two main methods of oxygen therapy, which are through hydrogen peroxide and ozone treatment:

a) Ozone treatment involves forcing oxygen through a metal tube carrying a 300-volt charge. Then a pint of blood drawn from the patient and placed into an infusion bottle. Ozone is then forced into the bottle and mixed so that the ozone molecules dissolve into the blood and, in so doing, destroy all lipid-envelope virus and many disease organisms, but leave the blood unharmed. Then the treated blood returned to the patient[2].

b) Hydrogen peroxide, when exposed to the blood or other body fluids containing the enzyme 'catalase' is chemically splits into oxygen and water. Many people have experienced the foaming effect produced by putting hydrogen peroxide on a wound; the foam is oxygen produced by the action of catalase on the hydrogen peroxide[3].

Each of oxygen therapy methods has some advantages and disadvantages. The point that should be note is that the real-time and online oxygen therapy is important to keep the patient in safe condition.

Pulse oximetry is a technology for noninvasively measurement of oxygen saturation, respiratory rate, etc. Using this technology the hypoxia can be detected before the patient show signs of becoming cyanotic[4]. Without pulse oximetry, it is hard to notice the patient has a decreased arterial oxygen saturation of hemoglobin $\left(\mathrm{Spo}_{2}\right)$ until the saturation is between $80-85 \%$. Pulse oximetry is simple to use and provides an immed iate, objective measure of arterial blood oxygen saturation.

However, the important question remains is that how can we use the pulse oximeter measurements in the best way to compensate the lack of oxygen in patients with different 
diseases. In fact, once the patient using the store oxygen of the body, the oxygen levelshould return to its regular level within minutes. Therefore, it is essential to use the oxygen at the times it shows low level. In this paper, we present a new method of online oxygenation, which has a reliable accuracy for various types of diseases.

\section{Pulse Oximeter Measurements}

Pulse oximetry is a non-invasive method of determining the percentage of hemoglobin $(H b)$ saturated with oxygen. Takuo Aoyagi, a biomedical engineer working for the Shimadzu corporation in Kyoto, Japan, in the early 1970, invented it $[5,6]$.

Knowing what percentage of the hemoglobin is saturated with oxygen is important when providing anesthesia or for determining the effectiveness of the respiratory system as well as helping in diagnosing various illnesses [7-9].

Pulse oximeter measures the absorption of red and infrared light passed through the patient's finger (ear lobe) by utilizing light sensors. Within the $\mathrm{Spo}_{2}$ sensor, light emitting diodes shine red and infrared light through the tissue.

The blood, tissue and bone at the application site absorb much of the light. However, some light passes through the extremity. A light-sensitive detector opposite the light source receives it.

Hemog lobin that is carry ing oxygen (oxy-hemoglobin) absorbs infrared wavelength of light and hemoglobin not carrying oxygen (de-oxy-hemoglobin) absorbs visible RED wavelength. Hemoglobin is a protein and the main component of red blood cells, and transports oxygen from the lungs, where oxygen tension (partial pressure of oxygen) $\mathrm{Spo}_{2}$ is high, to the tissues, where oxygen tension is low[10].

The sensor measures the amount of red and infrared light received by the detector and calculates the amount absorbed. Much of it is absorbed by tissue, bone and venous blood, but these amounts do not change dramatically over short periods.

Backg rounds such as fluid, tissue and bone are factored out of the measurement by monitoring the steady state absorption from bone, tissue, venous blood and arterial blood.

LEDs are used as the light source and are sequentially pulsed at a rapid rate. The amount of arterial blood does change over short periods due to pulsation (although there is some constant level of arterial blood). Because the arterial blood is usually the only light absorbing component, which is changing over short periods, it can be isolated from the other components.

During an arterial pulse, there is an increase blood volume and this AC component is used to calculate the absorption of oxy and de-o xy hemoglobin. The a mount of light received by the detector indicates the amount of oxygen bound to the hemoglobin in the blood.
From this data, the pulse oximeter does the mathematical calculations based on the Beer-Lambert Law to determine the percent oxygen saturation of the blood[11-12]:

$$
I=I_{0} e^{\varepsilon(\lambda) c d}
$$

where $I_{0}$ is the original intensity of the light, $\varepsilon(\lambda)$ is the extinction coefficient at a specific wavelength $\lambda$. In addition, $\mathrm{c}$ is the concentration of the substance absorbing the light, and $\mathrm{d}$ is the optical path length.

Pulse oximetry performed by placing a clip that contains two LEDs and the light sensor either onpatient's finger or on earlobe. One of the LEDs emits red light (600-700 $\mathrm{nm}$ ) and the other near infrared $(800-940 \mathrm{~nm})$.

Oxygen saturation $\left(\mathrm{Spo}_{2}\right)$ is defined, as the ratio of the amount of bound oxygen to the total oxygen capacity,equation (2), where $\left(\mathrm{Hbo}_{2}\right)$ is the concentration of oxy-hemoglobin, and $(R H b)$ is the concentration of de-oxy-hemoglob in. If the hemoglobin molecule is bound to oxygen then one has oxy-hemoglobin or $\left(\mathrm{Hbo}_{2}\right)$, and If the hemoglobin molecule is bound to carbon monoxide then one has carboxy-hemoglobin or ( $R b C o$ ). If the hemoglobin molecule is bound to nothing then one has de-oxy-hemoglo bin or $(R H b)$ or reduced hemoglobin. If the hemoglobin molecule has broken down then one has met-hemoglobin. These all have different spectra but here we concentrate on $\left(\mathrm{Hbo}_{2}\right)$ and $(\mathrm{RHb})$.

Oxygen saturation is calculated as a percent or fraction by[13-15]:

$$
\mathrm{Spo}_{2}=\frac{\mathrm{Hbo}_{2}}{\mathrm{Hbo}_{2}+\mathrm{Hb}} \times 100
$$

In healthy adults arterial oxygen saturation $\left(\mathrm{Spo}_{2}\right)$ is approximately $97 \%$. This depends on physiologicalparamet ers as well as on the oxygen partial pressure of the inspired air.

\section{New Method of Oxygenation}

A brief review of pulse oximeter and measuring the oxygen saturation of blood has been given. In fact, this brief review was necessary to explain our invention. The $\mathrm{Spo}_{2}$ measured by pulse oximeter is an accurate measurement of patient's oxygen saturation level, which in a normal status is about $97-98 \%$.

Usually after measuring the oxygen saturation, depending the $\mathrm{Spo}_{2}$ value and kind of disease physician prescribes a method to compensate the lack of oxygen.Finding outthe lack of oxygen on time, is a subject that physician and nurses are always worried about it.

Some people will experience symptoms such as shortness of breath or difficulty breathing and have a normal blood oxygen level. Others may experience no such symptoms and have a low blood oxygen level. Therefore, even though these symptoms are very important to watch and consider, it should not be decided to discontinue the oxygen based solely upon symptoms. The only way to know for sure is to have blood oxygen level checked with the oximetry test. 
To solve these problems and increase the accuracy coeffic ient of oxygenation, we have designed a mechanism that is working with a corresponding software and algorithm based on pulse oximeter measurements.

Figure 1 is representing the schema of system and figure 2 and 3 represent the algorithm and block diagram of the system respectively. The system consis ts of a simple pulse oximeter, an oxygen capsule, an A/D converter (to convert the digital $\mathrm{Spo}_{2}$ giving from pulse oximeter to the appropriate data for micro-programmer[16-18]), electric valve for controlling the oxygenation process, and a small LCD for showing the results and messages.

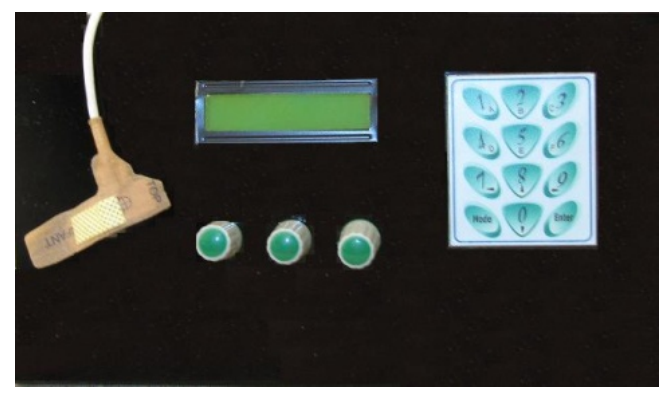

Figure 1. Schema of the system

\subsection{Software}

The use of mic rocontroller is common in every field even it can be use in design and fabrication of biomedical equipments. The89s51 (8051) microcontroller was used to develop the oxygenation system. The software of this system has the following capabilities:

a) Measuring the oxygen saturation of blood,

b) Comparing the oxygen saturation with the normal value,

c) Alarm to inform the health care staff when a problem like lack of oxygen or even over saturation occurs,

d) Controlling the electric valve that is connected to oxygen capsule or central oxygen of the hospital to give on-time oxygen to the patient.

\subsection{Algorithm of Working}

At first step, the kind of disease should be entered by keypad. Since each disease needs a specific amount of oxygen, (for example in chronic bronchitis the patient needs 2-3 lit/ $\mathrm{min}$, or in asthma 7-10 lit/min, and in cardiac ischemic 7-10 lit/min is needed) the kind of disease should first, be specified.

The device will compare the measured $\mathrm{Spo}_{2}$ with the normalvalue and display the result. That is, if the measured value is below the normal, alarm will also go off warn the health care staff. A warning message will be display on LCD and electric valve will be automatically open to allow the oxygen capsule start the oxygenation process.

Since $\mathrm{Spo}_{2}$ is measured continuously, as long as it did not reach to normal value, oxygenation process will be continued. After $\mathrm{Spo}_{2}$ gained to the normal value, the electric valve will be closed and oxygenation process will be stopped.
Moreover, this method can prevent the problem of over-saturation that sometimes occurred in other ways of oxygenation. If oxygenation process takes a long time, the over saturation message will represent on LCD and electric valve will be closed.

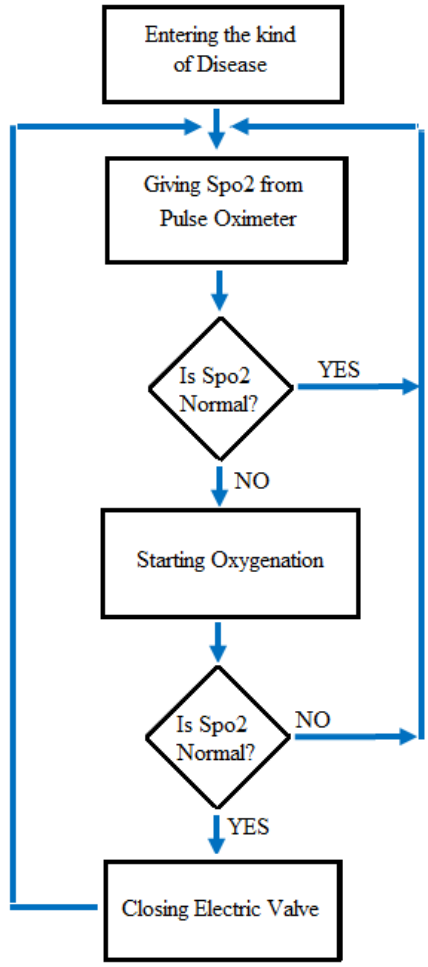

Figure 2. Algorithm of system

\section{Results}

We studied 10 patients; five COPD and five patients suffer of asthma (4 men and 6 women). Their meanage was $36.5 \pm 3.4$ years. None of the patients exhibited signs of oxygen toxicity during new method of oxygen therapy. The new method showseffective, safe, and on-time oxygenation for both types of examined dis eases.

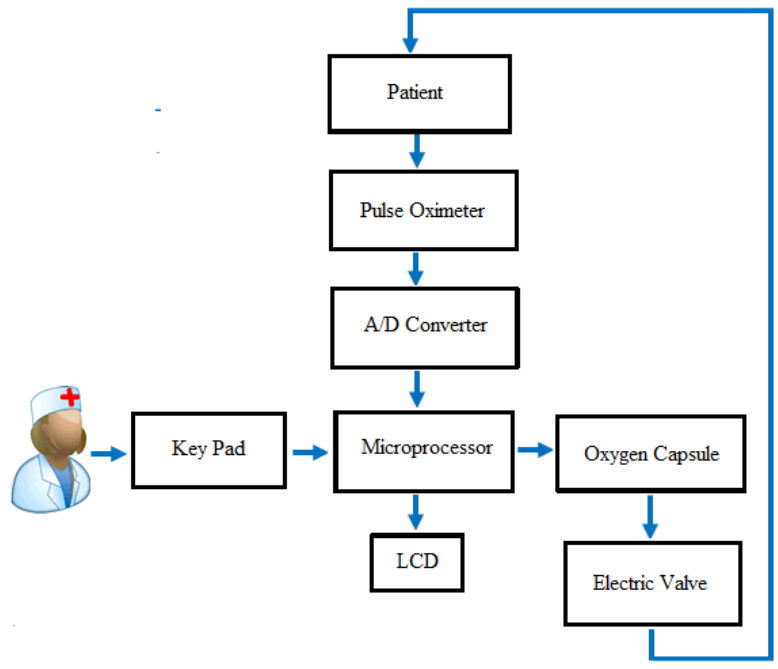

Figure 3. The block diagram of system 


\section{Conclusions}

Oxygen therapy helps many people function better and be more active. Although patients may need oxygen therapy for a long term, it does not have to limit their daily routine.

Portable oxygen units can make it easier to move around and do many daily activities. More people are using oxygen therapyoutside the hospital, permitting them to lead active, productive lives. People with asthma, emphysema, ch ronic bronchitis, occupational lung disease, lung cancer, cystic fibrosis, or congestive heart failure may use oxygen therapy at home.

Hospital environ ment could cause depression especially in patients with long confined period. Therefore, any methods such as oxygenation with pulse oximeter that can safe patients from hospitalization would be desirable.

Experimental results show that new oxygenation method coupled with pulse oximetry analys is is feasible, accurate, inexpensive and applicable to the clinical setting and home care services.Thepresented system in this paper could work with a 5-volt battery, so it could be a portable systemjust with adding a small oxygen capsule to the system.

Therefore, the homecare services, which are the most interesting topic in recent decade, could be practicable. Furthermore, by adding a pressure sens or to the oxygenation circuit, the safety of oxygenation especially in homecare services would be twice as much, because the pressure sensor could display the storage amount of oxygen in the capsule.With a simple calculation the capsule's volume could be estimated, so the user would be informed that the oxygen store would be finished for example during 2 hours.

In addition to health economizing the human time and energy spent, it will help the health care staff to increase the accuracy coefficient of oxygenation, especially when the number of patients is more than the staff. This method can avoid the over saturation that can occurs in other methods of oxygenation.

Our future aim is to equip the system, so that the data could be sending using Bluetooth wireless technology. The data can be stored and displayed locally for telemedicine applications. It can also transmit in real-time to a central monitoring centre over the internet, which would be well suited for remoterespiratory monitoring and home sleep apnea screening.

\section{REFERENCES}

[1] Thomas L. Croxton, William C. Bailey, "Long-term Oxy gen Treatment in Chronic Obstructive Pulmonary Disease: Recommendations for Future Research", American Journal of Respiratory and Critical Care Medicine, 174: 373-378, 2006.

[2] M. Bonettilo A. Fontana, and F. Albertini," CT-Guided Oxygen-Ozone Treatment for First Degree Spondylolis Thesis and Spondy loly sis", Acta Neurochir, 92: 87-92, 2005.
[3] Mark Zappi, Kenneth White,Huey-Min Hwang, Rakesh Bajpai, Mohammad Qasim," The Fate of Hy drogen Peroxide as an Oxygen Source for Bioremediation Activities within Saturated Aquifer Systems", Air \& Waste Manage. Assoc. 50:1818-1830, 2000.

[4] Esfandiar Akhavan Niaki,, Javad Chalipa, Elahe Taghipoor, "Evaluation of Oxygen Saturation by Pulse-Oximetry in Mouth Breathing Patients", Acta Medica Iranica, 48(1): 9-11, 2010 .

[5] Aoyagi T, Kishi M, Yamaguchi K, Watanabe S., "Improvem ent of an Earpiece Oximeter, 13th Annual Meeting of the Japan Society of Medical Electronics and Biological Engineering, Japan, pp 90-91, 1974.

[6] Makajima S, Hirai Y, Takase H, et al., "Performances of New Pulse Wave Earpiece Oximeter", Respir Circ, 23:41-45, 1975.

[7] A.T.Aldrich, "Non-invasive Blood Component Analyzer", U.S Patent Number :6046898, New York, 2000.

[8] May A, Huehns ER, "The Oxygen Affinity of Hemoglobin Hammersmith", British Joural of Haematolo gy, 30(2):185-95, 1975.

[9] Shinton NK, Thursby-Pelham DC, Williams HP., "Congenital Heinz-body Hemolytic Anemia due to Hemoglobin Hammersmith", Postgraduate Medical Journal, 45:629-632, 1969.

[10] Jubran A., “ Pulse Oximetry”, Crit Care, 3:R11-R17, 1999.

[11] Salyer JW. "Neonatal and Pediatric Pulse Oximetry". Respiratory Care Journal, 48:386-398, 2003.

[12] Severinghaus JW, "Some Personal Reflections", Anesth Analg Journal, 94: i-ii, 2002.

[13] Cote CJ, Goldstein EA, Cote MA, Hoaglin DC, Ryan JF., "A Single-Blind Study of Pulse Oximetry in Children", Anesthesiology, 68:184-188, 1988.

[14] Ralston AC, Webb RK, Runciman WB, "Pulse Oximetry: Instrumentation and Clinical Applications", Journal of Respiratory Care, 35:584-601, 1990.

[15] Clayton DG, Webb RK, Ralston AC, Duthie D, Runican WB, "A Comparison of the Performance of 20 Pulse Oximeters under Conditions of Poor Perfusion", Anaesthesia, 46:3-10, 1991.

[16] Carter BG, Carlin JB, Tibballs J, Mead H, Hochmann M., "Accuracy of Two Pulse Oximeters at Low Oxygen Hemoglobin-Saturation", Critical Care Medicine, 26:1128-1 133, 1998.

[17] Tsien CL, Fackler JC, "Poor Prognosis for Existing Monitors in the Intensive Care Unit", Critical CareMedicine, 25(4):614-619, 1997.

[18] Robertson FA, Hoffman GM., "Clinical Evaluation of the Effects of Signal Integrity and Saturation on Data Availability and Accuracy of Masimo SET and Nellcor N-395 Oximeters in Children”, Pediatric Anesthesia, 98:617-622, 2004. 